\title{
Management of Head-and-Neck Cancer during COVID-19 Crisis: A Medical Oncology Perspective
}

The ongoing COVID-19 pandemic is posing unique challenges to our health-care system. Oncology practices and protocols are adapting to the situation to help our patients. Head-and-neck squamous cell cancers are a huge burden in our country and need multidisciplinary care. This document shares some pragmatic ideas for medical oncologists on how to use systemic therapy for this disease in these challenging times.

The ongoing COVID-19 pandemic represents an unprecedented time in medicine and oncology in particular. Both current and former cancer patients are at a greater risk of being infected with COVID-19. ${ }^{[1-3]}$ The rate of "severe events" (defined as admission to the intensive care unit, the need for ventilation, or death) among cancer patients infected with COVID-19 exceeds that of the noncancer patients. ${ }^{[1-3]}$ The pandemic has added another layer of stress to cancer patients as there will be significant disruptions in their planned treatment protocols and consequent care, and things won't be normalized for quite sometime as per the statistical prediction of this pandemic.

In this "new normal" situation, health-care systems are being stretched to the maximum and the resources are being marshaled to provide good care to everyone, even though the care may look different than the conventional standards. Every specialty is thinking out of the box with innovative strategies adapted to the resource constraints and abiding by the norms of lockdown and social distancing. Our medical oncology practice has also been required to modify its pattern of care and bend around the established standards of care, to provide solutions to our patients. In many cases, these may be desperate measures without enough evidence backing them.

This novel rational model of care is based on the following key principles: (1) Does the benefit of a particular chemotherapy cycle exceeds the risk of morbidity and mortality from COVID-19? (2) Can we decrease the patient's hospital visits for the next few weeks of lockdown? (3) Can we reduce the number of emergency visits for oncology patients, as the emergency services are saturated and they may be triaged out? In simple words: "Do not overburden the overstretched health-care system."

Based on these basic principles, we have compiled some of our thoughts on how to manage chemotherapy in head-and-neck cancer patients in this unforeseen situation. These are neither guidelines nor consensus statements, rather our thoughts as a scientific association, to provide a helping framework for the oncology community to adapt to this unforeseen pandemic.
Head-and-neck cancers, especially squamous cell carcinoma (head-and-neck squamous cell carcinoma [HNSCC]), is the most common cancer among Indian males, accounting for $16.1 \%$ of cancer cases in them and accounted for 92,000 new cases in 2018. ${ }^{[4,5]}$ Worldwide, it is the $6^{\text {th }}$ leading cause of cancer, with an annual incidence of 550,000 and death of 300,000 cases. $^{[6]}$ HNSCCs are highly aggressive malignancies with high recurrence rates among locally advanced cancers even after curative therapy. ${ }^{[7]}$ Surgery plays a major role in oral cavity and tongue cancers, whereas concurrent chemoradiation is used for definitive therapy at most other sites. The role of chemotherapy is restricted to concurrent chemoradiotherapy (CTRT) in adjuvant/definitive settings and palliative chemotherapy for recurrent/metastatic settings.

\section{Adjuvant and Definitive Chemoradiotherapy}

Being highly aggressive cancers, it is imperative not to delay or compromise the adjuvant CTRT or definitive CTRT, wherever indicated. ${ }^{[8]}$ Accelerated radiotherapy may be considered to reduce the duration of treatment as daily travel to cancer centers during lockdowns is a challenge. Cleaning and sanitization of radiotherapy machines after each use is also recommended.

Only patients with absolute indications (positive margins and extranodal extension) for adjuvant CTRT should be offered the same. Most cisplatin-eligible patients will be fit without significant comorbidities, and hence, the benefits outweigh the risks. The 3-weekly cisplatin $\left(100 \mathrm{mg} / \mathrm{m}^{2}\right)$ may be discussed in fit patients to minimize visits to the day care, although this will not reduce the hospital footfall as the patients will continue their daily visits for radiation. At most centers, oncologists may be more comfortable with the weekly cisplatin of $40 \mathrm{mg} / \mathrm{m}^{2}$ schedule that requires fewer hospitalization for toxicity. In platinum-ineligible cases, cetuximab, carboplatin, and oral radiosensitizers such as capecitabine and hydroxyurea can be used with similar results. ${ }^{[9,10]}$

\section{Neoadjuvant Chemotherapy}

At many centers, elective surgeries for HNSCC are being postponed and as a time-buying procedure, increased demand for neoadjuvant chemotherapy (NACT) has emerged. In discussion with the multidisciplinary team, this needs to be explained to the patients and a shared decision should be made. 1-2 cycles of NACT, two-drug, 3-weekly regimen (e.g. paclitaxel + carboplatin/ cisplatin +5 -fluorouracil), which is less toxic than the three-drug regimen, may be used in this scenario. 
Oral metronomic therapy (OMT) may be a novel bridge to surgery in smaller lesions. However, this should be used very judiciously in consultation with the surgical team as for smaller lesions, surgery remains the best curative option. The use of any therapy in the neoadjuvant setting for HNSCC involves a risk of progression, which is not desirable. Useful regimens include tablet methotrexate $\left(15 \mathrm{mg} / \mathrm{m}^{2}\right)+$ celecoxib $^{[11-13]}$ or the triple-drug metronomic regimen of erlotinib + methotrexate + celecoxib. ${ }^{[14]}$ These regimens are effective with decent response rates and can halt stabilize tumor progression for at least a few weeks to tide over the crisis. These are oral regimens, can be taken at home without much-anticipated toxicity, perfectly complying with our concerns in the pandemic.

\section{Palliative Systemic Therapy}

Patients with fair performance status, tolerating palliative chemotherapy ( \pm cetuximab), may skip one/two sessions of chemotherapy after a mutual shared decision. For elderly patients ( $>70$ years) and those with significant comorbidities, single-agent cetuximab and oral metronomic chemotherapy can be given. Patients on immunotherapy alone may continue to receive, but at increased intervals (e.g. 4-weekly nivolumab instead of 2 weekly). ${ }^{[15]}$ Those on chemoimmunotherapy may drop chemotherapy and continue with immuno-oncology drugs for the next few weeks. Adverse effects among patients who receive immune checkpoint inhibitors (such as for severe myocarditis and pneumonitis) are more challenging to diagnose and might not be treated promptly, which might affect their survival. OMT has a significant role in this setting. It is best suited for the elderly and patients with comorbidities who have to be planned for a palliative regimen. Both double and triple metronomic regimens mentioned above can be used based on the physician's discretion. OMT may be used to tide over a few weeks in any patient awaiting palliative chemotherapy or radiotherapy. These ideas are summarized in Figure 1.
Growth factors, such as granulocyte colony-stimulating factor, may be used less stringently in elderly, frail patients or those with documented or anticipated toxicity in earlier cycles.

Oral hygiene, hand hygiene, and cough etiquettes are extremely important for HNSCC patients both on and off treatment, due to structural distortion of the aerodigestive tract, compromised mucocutaneous barrier, Ryle's tube, etc., Regular mouthwash with betadine and steam inhalation should be routinely advised and reiterated. A screening questionnaire for the symptoms of COVID-19 may be used.

\section{Telemedicine}

We must consider postponing routine follow-up visits for patients not on active treatment or use telemedicine. We need to interact with our patients and support and reassure them emotionally while maintaining social distancing using technology. Video-calling, virtual tumor boards using teleconferencing, emails, and chat messenger platforms are important tools to be frequently used for consultation, report viewing, etc., Importantly, the telemedicine facility should be arranged with local public or private cancer centers, minimizing patient's need to travel.

Examinations such as direct and indirect laryngoscopies are potential aerosol-generating procedures. Oncologists should take the utmost protection while performing such procedures.

For HNSCC, whether or not to postpone cancer treatment, a decision needs to be made on a patient-by-patient basis and according to the risk to the patient and the prevailing situation. Delays could lead to tumor progression and ultimately poorer outcomes. On the other hand, COVID-19 in an immunocompromised person can not only be more deadly, but it can also derail chemotherapy treatments

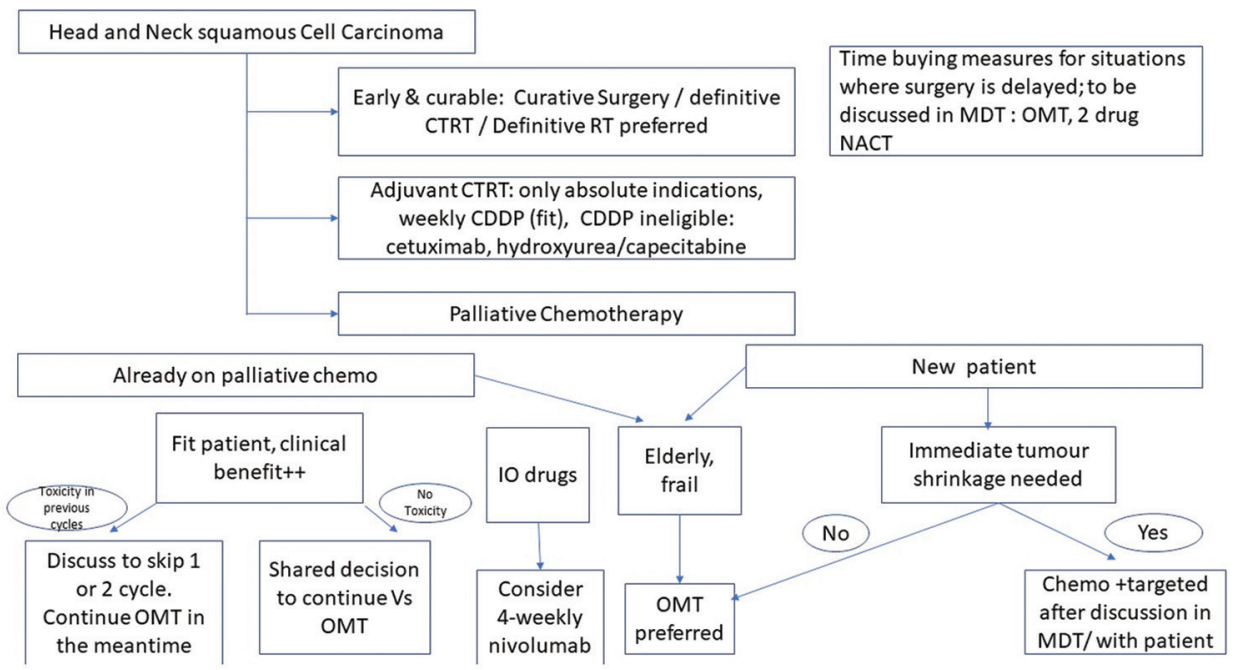

Figure 1: Algorithm for managing head-and-neck cancer chemotherapy during the COVID-19 pandemic 
and invite more risks for the patient and the health-care system. For each case, we should weigh what's more dangerous - leaving the house or missing a chemotherapy treatment.

Finally, the situation is evolving rapidly, and recommendations are likely to change as more evidence emerges specifically to oncology practice, patients with cancer, and COVID-19.

\section{Raja Pramanik', Priyanka Srivastava ${ }^{2}$, Atul Sharma ${ }^{1}$, Prashant Mehta ${ }^{3}$, Amol Patel ${ }^{4}$, Sainath Bhethanbhotla ${ }^{5}$, Bivas Biswas ${ }^{6}$, Atul Batra ${ }^{1}$, Vineet Govinda Gupta ${ }^{7}$, Chandan Krushna Das ${ }^{8}$, Shubh Mahendru ${ }^{9}$}

${ }^{I}$ Department of Medical Oncology, All India Institute of Medical Sciences, New Delhi, India, ${ }^{2}$ M.S.Patel Cancer Center, Shree Krishna Hospital and Medical Research Centre, Karamsad, Gujarat, India, ${ }^{3}$ Department of Medical Oncology, Asian Institute of Medical Sciences, Faridabad, Haryana, India, ${ }^{4}$ Malignant Diseases Treatment Centre, Army Hospital Research and Referral, New Delhi, India, ${ }^{5}$ Department of Medical Oncology, Care Cancer Institute, Hyderabad, Telangana, India, ${ }^{6}$ Department of Medical Oncology, Tata Medical Center, Kolkata, West Bengal, India, 'Department of Medical Oncology, Artemis Hospital, Gurgaon, Haryana, India, ${ }^{8}$ Regional Cancer Centre, PGI-MER, Chandigarh, India, ${ }^{9}$ Department of Surgical Oncology, Ivy Hospital, Ajitgarh, Punjab, India

Address for correspondence: Dr. Raja Pramanik, Department of Medical Oncology, All India Institute of Medical Sciences, New Delhi, India.

E-mail:drrajapramanik@gmail.com

Submitted: 04-Apr-2020

Revised: 17-Apr-2020

Accepted: 19-Apr-2020

Published: 08-May-2020

\section{References}

1. Onder G, Rezza G, Brusaferro S. Case-Fatality Rate and Characteristics of Patients Dying in Relation to COVID-19 in Italy [published online ahead of print, 2020 Mar 23]. JAMA. 2020;10.1001/jama.2020.4683. doi:10.1001/jama.2020.4683.

2. Wang D, Hu B, Hu C, Zhu F, Liu X, Zhang J, et al. Clinical Characteristics of 138 Hospitalized Patients With 2019 Novel Coronavirus-Infected Pneumonia in Wuhan, China [published online ahead of print, 2020 Feb 7]. JAMA. 2020;e201585. doi:10.1001/jama.2020.1585.

3. Wang H, Zhang L. Risk of COVID-19 for patients with cancer. Lancet Oncol 2020;21:e181.

4. 356-India-Fact-Sheets. Available from: https://gco.iarc.fr/today/ data/factsheets/populations/356-india-fact-sheets.pdf. [Last accessed on 2020 Mar 29].

5. Sharma S, Satyanarayana L, Asthana S, Shivalingesh KK, Goutham BS, Ramachandra S. Oral cancer statistics in India on the basis of first report of 29 population-based cancer registries. J Oral Maxillofac Pathol 2018;22:18-26.

6. Poddar A, Aranha R, Royam MM, Gothandam KM, Nachimuthu R, Jayaraj R. Incidence, prevalence, and mortality associated with head and neck cancer in India: Protocol for a systematic review. Indian J Cancer 2019;56:101-6.

7. Denaro N, Merlano MC, Russi EG. Follow-up in head and neck cancer: Do more does it mean do better? A systematic review and our proposal based on our experience. Clin Exp Otorhinolaryngol 2016;9:287-97.

8. Kutikov A, Weinberg DS, Edelman MJ, Horwitz EM, Uzzo RG, Fisher RI. A War on Two Fronts: Cancer Care in the Time of COVID-19 [published online ahead of print, 2020 Mar 27]. Ann Intern Med. 2020;M20-1133. doi:10.7326/M201133 .

9. Vokes EE, Panje WR, Schilsky RL, Mick R, Awan AM, Moran WJ, et al. Hydroxyurea, fluorouracil, and concomitant radiotherapy in poor-prognosis head and neck cancer: A phase I-II study. J Clin Oncol 1989;7:761-8.

10. Salama JK, Haraf DJ, Stenson KM, Blair EA, Witt ME, Williams R, et al. A randomized phase II study of 5-fluorouracil, hydroxyurea, and twice-daily radiotherapy compared with bevacizumab plus 5-fluorouracil, hydroxyurea, and twice-daily radiotherapy for intermediate-stage and T4N0-1 head and neck cancers. Ann Oncol 2011;22:2304-9.

11. Patil VM, Noronha V, Joshi A, Nayak L, Pande N, Chandrashekharan A, et al. Retrospective analysis of palliative metronomic chemotherapy in head and neck cancer. Indian $\mathrm{J}$ Cancer 2017;54:25-9.

12. Patil VM, Noronha V, Joshi A, Banavali SD, Muddu V, Prabhash K. Preoperative chemotherapy and metronomic scheduling of chemotherapy in locally advanced oral cancers. Oncology 2016;91 Suppl 1:35-40.

13. Sultania M, Deo SV, Shukla NK, Sharma A, Sahu RK, Bhasker S. Neoadjuvant metronomic chemotherapy in locally advanced head and neck cancers - A feasibility study. Eur J Cancer 2017;72:S106.

14. Patil VM, Noronha V, Joshi A, Dhumal S, Mahimkar M, Bhattacharjee A, et al. Phase I/II study of palliative triple metronomic chemotherapy in platinum-refractory/early-failure oral cancer. J Clin Oncol 2019;37:3032-41.

15. Long GV, Tykodi SS, Schneider JG, Garbe C, Gravis G, Rashford $\mathrm{M}$, et al. Assessment of nivolumab exposure and clinical safety of $480 \mathrm{mg}$ every 4 weeks flat-dosing schedule in patients with cancer. Ann Oncol 2018;29:2208-13.

This is an open access journal, and articles are distributed under the terms of the Creative Commons Attribution-NonCommercial-ShareAlike 4.0 License, which allows others to remix, tweak, and build upon the work non-commercially, as long as appropriate credit is given and the new creations are licensed under the identical terms.

For reprints contact:WKHLRPMedknow_reprints@wolterskluwer.com

\begin{tabular}{|l|l|}
\hline \multicolumn{2}{|c|}{ Access this article online } \\
\hline Quick Response Code: & Website: \\
& www.ijmpo.org \\
\cline { 2 - 2 } & DOI: \\
\hline
\end{tabular}

How to cite this article: Pramanik R, Srivastava $P$, Sharma A, Mehta $P$, Patel A, Bhethanbhotla S, et al. Management of head-and-neck cancer during COVID-19 crisis: A medical oncology perspective. Indian J Med Paediatr Oncol 2020;41:153-5. 